\title{
Evaluation of the effectiveness of the use of heat pumps of the "water-water" type in the wastewater disposal system of the building
}

\author{
Sergey Kuzmin and Leonid Kudelkin \\ Federal State Budgetary Educational Institution of Higher Education "Angarsk State Technical \\ University", 665835 Angarsk, Russia
}

\begin{abstract}
The paper presents the results of the analysis of the effectiveness of the use of heat pumps of the "water-water" type for the extraction of thermal energy from the wastewater of wastewater disposal systems of residential buildings. A system of equations characterizing the operation of heat pumps in the system - "drains-heat consumption" - is obtained. The thermal potential of the wastewater and the cost of the extracted energy were estimated.
\end{abstract}

\section{Introduction}

The consumption of thermal energy by the engineering systems of the building (heating, ventilation, hot water supply) is transit in nature - the working environment (indoor air, supply ventilation, hot water) is heated to the design temperature and then removed from the building, irretrievably giving excess heat to the environment. Thus, the energy leaving the building is a potential source of heat for secondary use.

Currently, the most widespread systems for extracting heat from the outgoing ventilation air by means of various heat exchangers [1] or air-to-water heat pumps [2-4]. However, as the experience of operating such devices and the analysis of work shows. [5-7], the efficiency of these systems depends on the changing state of the heat-containing environment - outdoor air.

Water-to-water heat pumps have higher thermodynamic efficiency and operational stability [4]. But their practical application is limited by the presence of a source of natural water near the object and the difficulty with the laying and operation of heat exchangers in water bodies. At the same time, almost every person in operation has a source of relatively warm water - a drain liquid. With a relatively stable flow rate and temperature throughout the year, wastewater can prove to be an effective source of heat energy for heat pumps and a useful component in the overall balance of heat generators for a building.

\section{Materials and methods}

In this paper, the thermal potential of wastewater in buildings is estimated. The possible effect of the extraction of thermal energy from the waste fluid of residential buildings by heat pumps was determined and the cost of this method of heat production was estimated. The 
technique is based on the use of mathematical models of the characteristics of technical devices of engineering equipment, statistical models of economic indicators of equipment.

\section{Results and Discussion}

The reserve amount of heat that can be extracted from the wastewater depends on its amount for the calculation period and temperature potential. The composition of effluents in residential and public buildings is determined by economic and fecal fluids. It is obvious that the utilization of thermal energy is rational to produce from economic (or production) effluents. Given that the proportion of fecal fluid does not exceed $10 \%$ of the total flow, the remaining amount of wastewater can be considered as a potentially heat-containing medium.

It is known that the mode of water consumption in the building, although subject to daily regularity, but the hourly (and instantaneous - second) is significantly uneven. But bearing in mind the availability of technical possibilities for the use of regulating tanks in the wastewater disposal system, we use hourly averages to determine the amount of extracted thermal energy of wastewater:

$$
W_{w}=Q_{w} \cdot \tau
$$

where is $Q_{w}$ - average hourly power of heat extracted from wastewater, kW;

$\tau$ - period of operation of the heat pump, hour/year.

Thermal power of wastewater $Q_{w}[W]$ is determined by its number and potential differenties of the medium up to $t_{w 1}$ and after $t_{w .3}$ heat pump:

$$
Q_{w}=0,28 \cdot G_{w} \cdot \rho_{\varpi} \cdot c_{\varpi} \cdot\left(t_{w 1}-t_{w 3}\right),
$$

where is $G_{w}$ - estimated amount of wastewater removed by sewerage systems, $m^{3} /$ hour

$c_{w}$ and $\rho_{w}$ - accordingly heat capacity $\left(k J / \kappa g^{\circ} C\right)$ and water density $\left(\kappa g / \mathrm{M}^{3}\right)$

at temperature $t_{w .1}$.

Estimated average hourly amount of wastewater $G_{w}$ will be determined from the daily rate of water consumption of cold $g_{c}$ and hot water $g_{h}$ and the number of consumers in the building $U$ :

$$
G_{\kappa}=\frac{U}{24}\left(0,9 \cdot g_{c}+g_{h}\right)
$$

The number of consumers is represented through the rate of usable area per inhabitant $f_{n}$ and geometric characteristics of the building:

$$
U=\frac{A_{\Pi}}{f_{\Pi}}=\frac{S * k_{\Pi} * n_{\ni m}}{f_{\Pi}}=\frac{V^{*} k_{\Pi} * n_{э m}}{H^{*} f_{\Pi}}=\frac{V^{*} k_{\Pi} * n_{\ni m}}{h_{э m} * n_{э m} * f_{\Pi}}=\frac{V^{*} k_{\Pi}}{h_{\ni m} * f_{\Pi}},
$$


where is $A_{\Pi}$ - total usable area in the building, $m^{2} ; S$ - horizontal projection area of the building, $m^{2} ; k_{\Pi}$ - a factor that determines the share of usable space in a building in its total area; $n_{э m}$ - number of storeys of the building; $V$ - useful volume of the building, $\mathrm{m}^{3}$; $H$ - height of the useful volume of the building, $m ; h_{э m}$ - floor height, $m$.

The weighted average temperature of the effluents will be determined from the calculated temperatures of the hot $t_{h}$ and cold $t_{c}$ water and the ratio of water consumption rates:

$$
t_{w 1}=\frac{g_{h} t_{h}+0,9 \cdot g_{c} t_{c}}{g_{h}+g_{c}}
$$

Taking into account (10-13) the average estimated hourly amount of heat to be recycled (W) can be determined by the expression:

$$
Q_{w}=\frac{0,28}{24}\left(g_{h}+0,9 \cdot g_{c}\right) \frac{V^{*} k_{\Pi}}{h_{\ni m} * f_{\Pi}} \cdot \rho_{\varpi} \cdot c_{\varpi}\left(\frac{0,9 \cdot g_{c} t_{c}+g_{h} t_{h}}{0,9 \cdot g_{c}+g_{h}}-t_{w 3}\right) .
$$

The graphs (Figure.1) show the calculated values of the heat and energy reserve in the waste fluid at different rates of water consumption and temperature conditions in relation to the corresponding values of the heating system of buildings, located in a "moderately cold" climatic area with a design temperature for heating $t_{c o}=-35^{\circ} \mathrm{C}$ and "moderate" with design temperature $t_{c o}=-25^{\circ} \mathrm{C}$.

a)

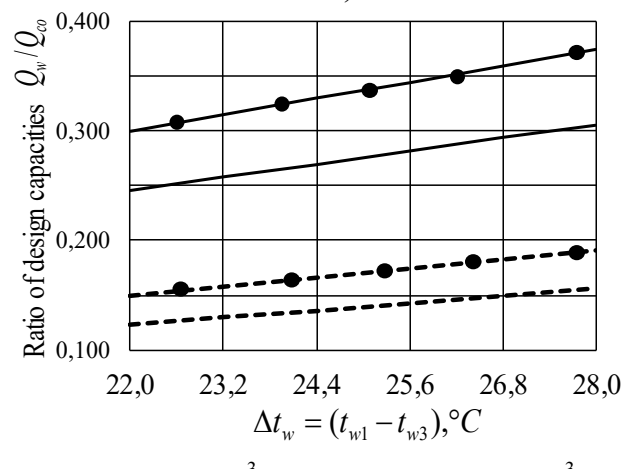

--- $\mathrm{q}_{\mathrm{c}}=0,1 \mathrm{~m}_{3}^{3} /$ people's day. $\mathrm{q}_{\mathrm{c}}=0,06 \mathrm{~m}_{3}^{3} /$ people's day;

$-\mathrm{q}_{\mathrm{c}}=0,18 \mathrm{~m}^{3} /$ people's day. $\mathrm{q}_{\mathrm{c}}=0,1 \mathrm{~m}^{3} /$ people's; day; b)
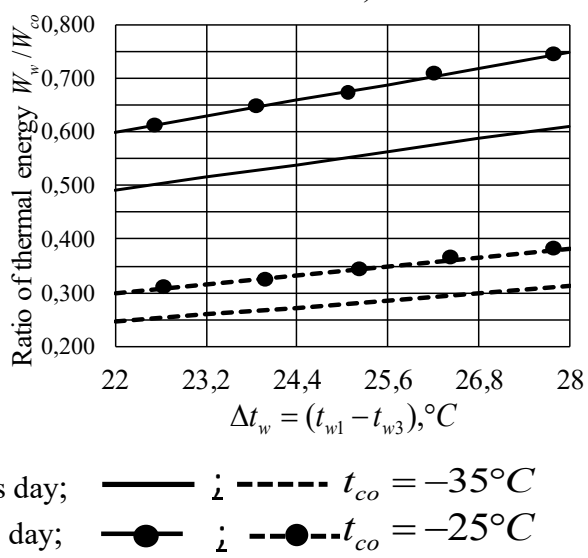

Fig. 1. Ratios between the capacity of the potential of the effluents and the design capacity of the heating system $Q_{w} / Q_{c o}$ - a) and the amount of heat contained in the runoff and the heat requirement for heating the building $W_{w} / W_{c o}$ for the billing period - b).

Calculations show that the reserve of thermal power of the wastewater reaches from 12 $\mathrm{W}$ to $25 \mathrm{~W}$ per $1 \mathrm{~m}^{2}$ of the heated room, which is a significant share of the design capacity for heating the building: from 0.15 to 0.3 for "cold" areas and even up to $40 \%$ for "moderate". An even greater proportion of wastewater heat in the necessary energy for heating buildings, which can reach from $25 \%$ to $60 \%$ for "cold" areas and even $80 \%$ for temperate climates. This effect is achieved due to the relatively constant reserve of the energy capacity of the 
effluents during the year with a changing heating demand during the heating period.

Thus, the analysis of the thermal energy reserve of the waste fluid provides the basis for considering this low-potential environment as a heat source for the engineering systems of the building. The problem lies not so much in the technical implementation of the process of heat extraction with an increase in temperature potential, but in the possible costs and, accordingly, the profitability of this method of energy supply. The efficiency of using wastewater as an energy source will be estimated by the cost of the heat extracted $C_{W}$ (c.u./MW*h):

$$
C_{W}=\frac{C_{Q}}{W_{w}}=\frac{C_{T H} \cdot k_{T H} \cdot K_{M}+C_{N}}{W_{w}},
$$

where is $C_{T H}$ - cost of heat pump, c.u.;

$k_{T H}$ - coefficient of self-sufficiency of heat pump, year ${ }^{-1}$;

$C_{N}$ - heat pump operating costs per year, c.u./year;

$K_{M . T H}$ - correction factor, taking into account the cost of installation work;

$W_{w}$ - the amount of heat extracted from the effluents during the billing period, $\mathrm{kW}^{*} \mathrm{~h} /$ year.

The cost of a heat pump is represented by dependence on its calculated (nominal) thermal performance $Q_{T H . o}$ :

$$
C_{T H}=c_{q} * Q_{T H . o}
$$

where is $C_{q}$ - cost indicator (unit cost) attributed to the unit of heat power of the pump, c.u./kW.

As the analysis of prices for water-to-water heat pumps on the market shows, their cost varies widely from different manufacturers. $[8,9]$. For the universality of the analysis, the cost of equipment is expressed in conventional units (c.u.) and is presented in figure 2.

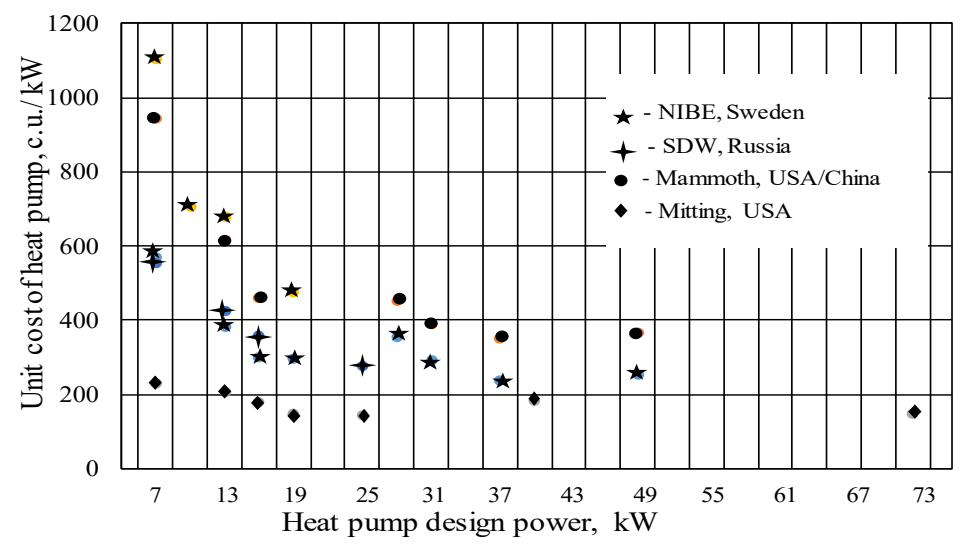

Fig. 2. Distribution of unit cost of heat pumps Cq, c.u./kW by design power $Q_{T H . o}, \mathrm{~kW}$. 
This distribution defines expressions for the maximum $c_{q}^{\wedge}$ and minimum $c_{q}^{\vee}$ unit cost values

$$
c_{q}^{\wedge}=2100 \cdot\left(Q_{T H}^{m}-4\right)^{-0,531} ; \quad c_{q}^{\vee}=248 \cdot\left(Q_{T H}^{m}-4\right)^{-0,147}
$$

Installation costs are usually correlated with the cost of equipment with some reducing adjustment for more expensive systems and range from $50 \%$ to $80 \%$ of $C_{T H}$. Therefore, the coefficient $K_{M . T H}$ in (7) can be represented as:

$$
K_{M . T H}=1,825-0,004 \cdot Q_{T H . o}
$$

The operating costs of the heat pump will be determined through the current electrical capacity $N_{T H . t}$ and opening hours $\tau_{T H}$ :

$$
C_{N}=c_{N} \cdot W_{K}=c_{\text {эл }} \cdot N_{T H . t} \cdot \tau_{T H},
$$

where is $c_{N}$ - electricity tariff, c.u. $/ \mathrm{kW}^{*} \mathrm{~h}$.

Current electrical capacity of the heat pump compressor $N_{T H . t}$ depends on the rated power $N_{T H . o}$, temperature of heat-containing medium $t_{w 1}$ and heat conversion efficiency:

$$
N_{\text {TH.t }}=N_{\text {TH.o }} \cdot\left[1-0,054 \cdot \ln \left(0,2 \cdot t_{w 1}+1\right)\right] .
$$

The ratio of the rated heat power of the pump to the (installation) power of the compressor shows the design efficiency of heat energy conversion $C O P_{o}$. For water-to-water heat pumps, the design conditions usually correspond to the temperatures of the heat-containing medium $t_{w 1}=20^{\circ} \mathrm{C}$ and secondary coolant $t_{w 2}=60^{\circ} \mathrm{C}$ and on average $C O P_{o}=4,2$. Current value $C O P_{t t}$ decreases compared to nominal with decrease $t_{w 1}$ and increases as the temperature decreases at the outlet of the system $t_{w 2}$. This ratio can be estimated by the expression (8):

$$
\begin{aligned}
& \operatorname{COP}_{t t}=\left(0,0021 \cdot t_{w 2}-0,2 \cdot t_{w 2}^{2}-5,38\right) \cdot \ln \left(0,2 \cdot t_{w 1}+1\right)- \\
& -0,0028 \cdot t_{w 2}^{2}+0,232 \cdot t_{w 2}-1,048
\end{aligned}
$$

Accordingly, the current electrical capacity of the compressor will be:

$$
N_{T H . t}=\frac{Q_{T H . o}}{C O P_{t t}} \cdot\left[1-0,054 \cdot \ln \left(0,2 \cdot t_{w 1}+1\right)\right]
$$

Amount of heat $W_{w}$, extracted from effluents during the billing period $\tau_{T H}$ under stable conditions, will be determined from (15):

$$
W_{w}=Q_{w} \cdot \tau_{T H} .
$$

Thus, equations (6-15) make up a system that allows solving the problem. 
As an illustration of the obtained model of profitability of the method of extracting heat from household wastewater, consider the effectiveness of the method on the example of a residential apartment building. For a residential building with a high degree of improvement, equipped with all the basic sanitary appliances (bath with shower, sink, sink in the kitchen, toilet), the rate of water consumption per inhabitant is hot water $\left(g_{h}=120 l / d a y\right)$, cold $\left(g_{c}=180 l / d a y\right)$ [10]. Maximum cooling temperature of wastewater $t_{w 3}$ accept not lower than $+5^{\circ} \mathrm{C}$. Then at the height of the floor of the building $h_{э m}=3 m$, utility factor $k_{\Pi}=0,8$ and usable area $f_{\Pi}=18 m^{2} /$ peopl formula (6) will take the form:

$$
Q_{w}=0,117 \cdot V \cdot\left(t_{c}+0,74 \cdot t_{h}-8,7\right)
$$

Taking the value $Q_{w}$ as the rated power of the heat pump $Q_{T H . o}$ let's analyze the possible cost of energy extracted by a heat pump from wastewater under various conditions.

On figure 3 shows the dependence of the cost of heat obtained from wastewater on the temperature of cold water $t_{c}$ at hot water temperatures $t_{h}=60^{\circ} \mathrm{C}$ a) and $t_{h}=40^{\circ} \mathrm{C}$ в) for a building with a volume of $1000 \mathrm{~m} 3$ and the use of high cost heat pumps (9). A feature of the behavior of the objective function is a relatively weak dependence not only on the temperatures of the initial media, but also on the level of potential of the energy produced. With an increase in the temperature of cold water on $10^{\circ} \mathrm{C}$ (three times), and the temperature of hot water on heat cost is reduced by less than $1 \%$. Also below expectations, the effect of increasing the efficiency of the heat pump is manifested while reducing the level of potential of the heat produced.c $t_{w 2}=60^{\circ} \mathrm{C}$ before $t_{w 2}=40^{\circ} \mathrm{C}$ the cost of energy is reduced by $(1-2) \%$.

The obtained values show that with a relatively short period of self-sufficiency and low cost of electricity, the cost of the extracted heat is obtained about 4 times higher than the selling price, for example, the energy system of JSC «Irkutskenergo» under any initial conditions.

a)

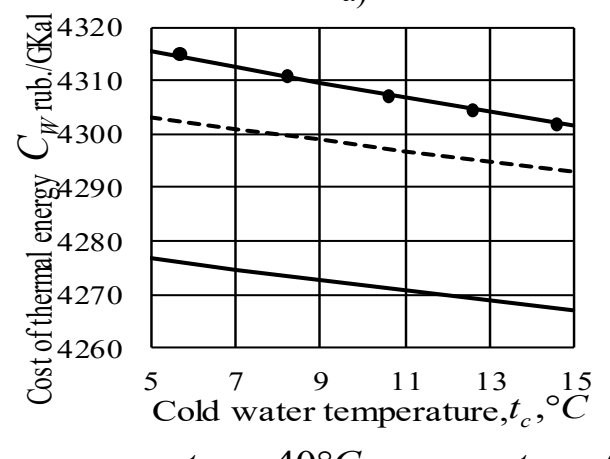

b)

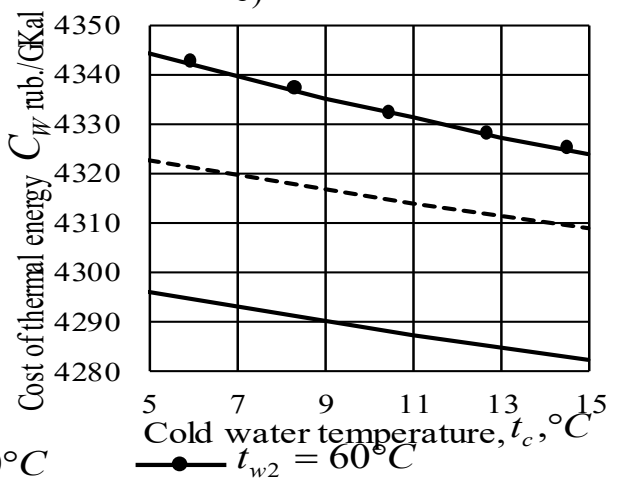

Fig. 3. The cost of thermal energy for the period of self-sufficiency of 5 years and the cost of electricity is 1.5 rubles $/ \mathrm{kW} * \mathrm{~h}$.

For more "expensive" energy systems, the situation is somewhat improving. With an electricity tariff of 5 rubles / $\mathrm{kWh}$ and 10 rubles / $\mathrm{kWh}$, the cost of heat, although it increases on average to 5050 rubles / Gcal and 6200 rubles / Gcal, respectively, which is only $2-2.5$ times higher than the selling price («Mosenergo» JSC). The high cost of thermal energy is 
determined primarily by the large capital costs of the equipment and the short payback period adopted. If it is permissible to increase this figure to 10 years, then the efficiency is significantly improved (Fig. 4) and the cost of heat produced by the heat pump, although it remains higher than in the district heating system, but this excess is not more than (40-50)\%. Although under this condition, the mode of operation of the pump has little effect on the overall economic efficiency of the system.

a)

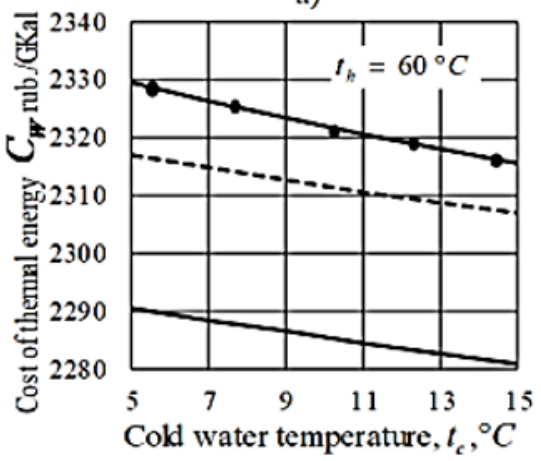

b)

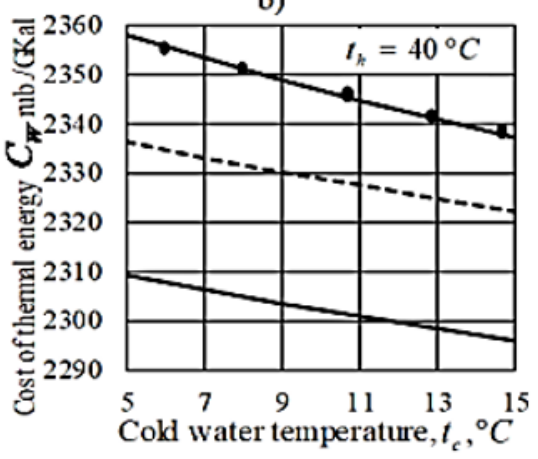

$\longrightarrow t_{w 2}=60^{\circ} \mathrm{C}$

Fig. 4. The cost of thermal energy for the period of self-sufficiency of 10 years and the cost of electricity is 1.5 rubles $/ \mathrm{kW} * \mathrm{~h}$.

An even more significant reduction in cost is achieved for a set of cheaper equipment. The distribution of prices for heat pumps (figure 1) shows a significant price difference, especially in the range of nominal capacity from 4 to $30 \mathrm{~kW}$. Without considering the consumer qualities of the equipment and focusing on the lower price level, it can be shown that the extraction of heat from the waste fluid is more profitable than traditional methods. On figure 5 shows the cost of heat production when using cheap equipment under the conditions already considered (figure 2,3). For buildings of small volume (up to $2000 \mathrm{~m} 3$ it is possible to reduce the cost of the heat pump by about 5 (!) times. And this immediately increases the efficiency of heat pumps, even with a short payback period.

a)

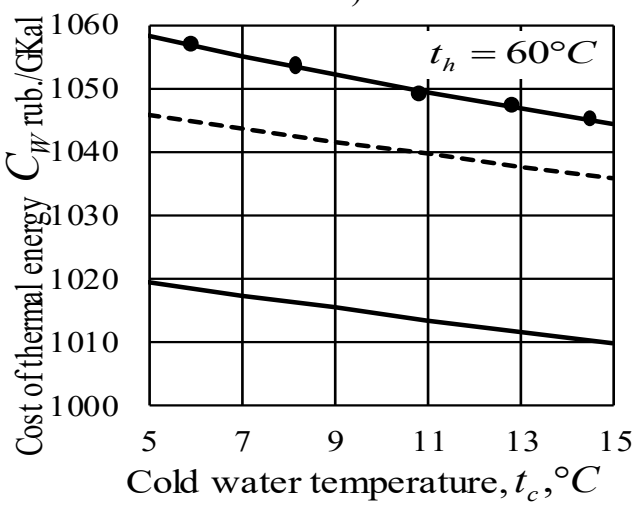

b)

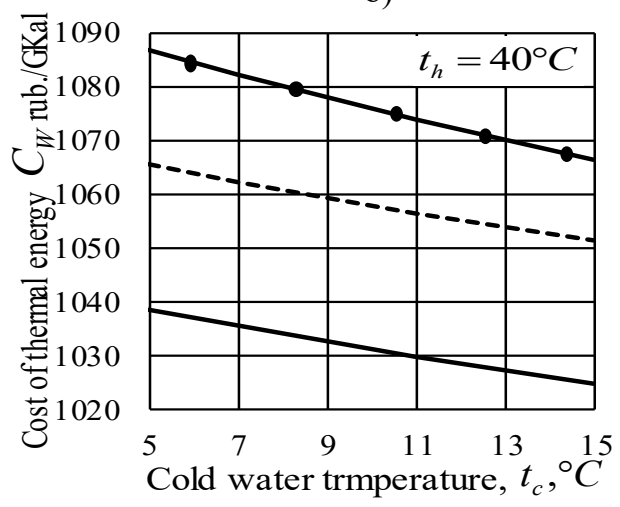

Fig. 5. The cost of thermal energy for the period of self-sufficiency of 5 years and the cost of electricity is 1.5 rubles $/ \mathrm{kW}^{*} \mathrm{~h}$ at the lower level of prices for equipment. 
The consequence of a decrease in the unit cost of heat pumps with an increase in nominal capacity is a noticeable decrease in the cost of energy produced with an increase in the volume of the building. On Fig. Fig. 6 shows the change in the cost of heat for buildings of different volumes relative to the cost of heat for a building of $1000 \mathrm{~m} 3$ under the same conditions.

A 7-factor increase in volume leads to a decrease $C_{W}$ almost $65 \%$ almost regardless of the temperature of cold water, $t_{c}=+15^{\circ} \mathrm{C}$ with "cheap" electricity. The influence of cold water temperature is noticeably is noticeably manifested at high electricity tariffs (figure 6 b).

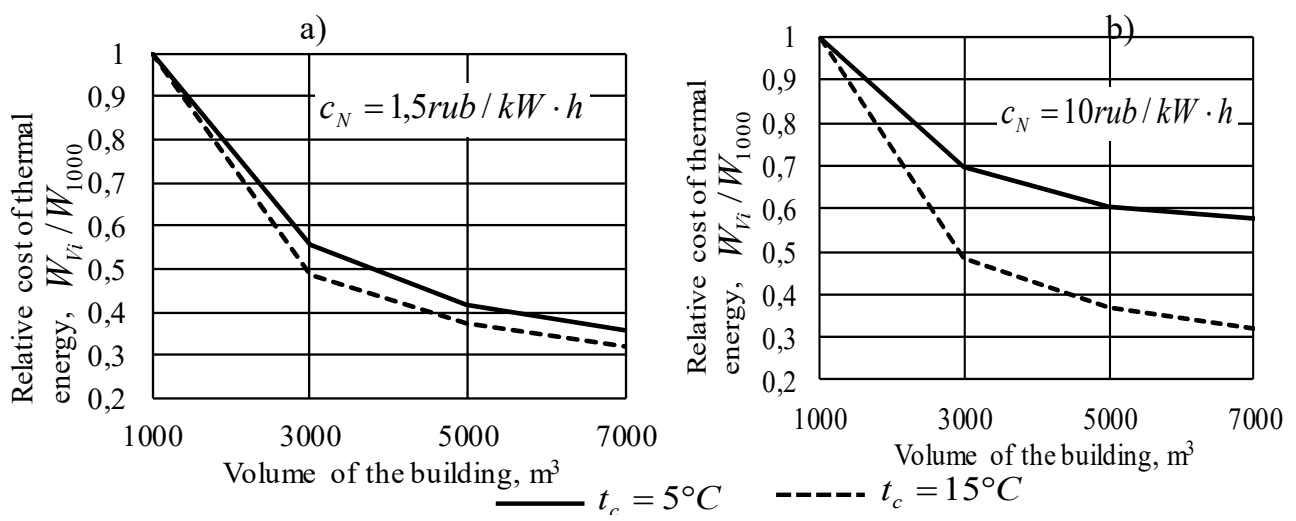

Fig. 6. The relative cost of thermal energy for the period of self-sufficiency of 5 years and the cost of electricity 1.5 rubles / $\mathrm{kW}^{*} \mathrm{~h}-\mathrm{a}$ ) and 10 rubles $/ \mathrm{kW}^{*} \mathrm{~h}-\mathrm{b}$ ).

Although with the inflated cost of electricity for large buildings, the use of a heat pump can be economically beneficial, especially if you focus on a long payback period. At the same time, the cost of thermal energy may be significantly lower (by 25-35\%) of the tariff of the energy system.

\section{Conclusion}

The analysis shows that the use of water-to-water heat pumps using wastewater as a heat source can be quite a competitive option for a heat generator in building heating systems. Under certain conditions, the cost of energy extracted from wastewater is either comparable or even lower than the tariffs set by regional heat supply organizations.

Of course, the implementation of such a scheme will require a change in the design of the wastewater disposal system associated with the separation of fecal and household wastewater, the accumulation of the latter in an intermediate tank, etc. But these changes do not seem fundamentally feasible even in existing buildings, and when designing new facilities are quite feasible, especially since most buildings provide for two or more sewer risers within one apartment.

The increased cost of thermal energy in small buildings or with the assigned low payback periods of the equipment also does not seem critical. Not to mention the environmental friendliness of this method of heat production, it should be noted that even under adverse conditions, the cost of recoverable energy (4300-6200) rubles / Gcal is not higher than the cost of energy generated by other renewable sources - wind generators, solar stations.

The identified dependencies that establish the efficiency of heat pumps in the system under consideration showed a weak effect of the variability of the state of the environment and the temperature potential of the generated energy on its cost. This suggests a wide 
possibility of applying this scheme in various objects.

Thus, the above calculations allow us to conclude about a certain prospects of this method of using heat pumps of the "water-water" type. Of course, this requires the behavior of work to change the design of wastewater disposal systems of buildings and even new planning solutions for objects, but given the results obtained and the relevance of the energy saving aspect, such efforts seem quite justified.

\section{References}

1. Designer's Handbook. Internal sanitary devices part 3. Ventilation and air conditioning, 416 (1992)

2. S. K. Luneva, A.S. Chistovich and I. H. Emirov, Tech and Technology Service Problems Magazine, 2, 15 (2013)

3. Y. V. Petin New generation of heat pumps for heating purposes and efficiency of their use in Russia, 175 (2004)

4. Heat pumps in modern industry and municipal infrastructure. Information and methodical edition, 204 (2016)

5. S. I. Kuzmin and N. A. Ivshina, IOP CS: MSaE, 880 (2020)

6. S. I. Kuzmin and A.A. Zelenina, IOP CS: MSaE, 880 (2020)

7. S. I. Kuzmin S I and A. V. Zateeva, IOP CS: MSaE, 880 (2020)

8. Price list for heat pumps Meeting, https//solar-dom.com

9. Price list for heat pumps Mammoth, https:// mammoth-russion.ru

10. SP 30.13330. 2016 Internal water supply and sewerage (2016) 